\title{
Dynamic characteristics of Semi-active Hydraulic Engine Mount Based on Fluid-Structure Interaction FEA
}

\author{
Jiande Tian $^{2}$, Xue Jiang ${ }^{1}$, Guozheng Liu ${ }^{1}$, Wenku Shi ${ }^{1, a}$,Baoquan Liu ${ }^{2}$, Meiqin Ma ${ }^{2}$ \\ ${ }^{1}$ College of Automotive Engineering, Jilin University, China \\ ${ }^{2}$ Hengshui Rubber Co.,Itd, Hengshui, China
}

\begin{abstract}
A kind of semi-active hydraulic engine mount is studied in this paper. After careful analysis of its structure and working principle, the FEA simulation of it was divided into two cases. One is the solenoid valve is open, so the air chamber connects to the atmosphere, and Fluid-Structure Interaction was used. Another is the solenoid valve is closed, and the air chamber has pressure, so Fluid-Structure-Gas Interaction was used. The test of this semi-active hydraulic engine mount was carried out to compare with the simulation results, and verify the accuracy of the model. Then the dynamic characteristics-dynamic stiffness and damping angle were analysed by simulation and test. This paper provides theoretical support for the development and optimization of the semi-active hydraulic engine mount.
\end{abstract}

\section{Introduction}

Because the vibration and noise of car mainly comes from the engine, the engine mount performance will directly affect the vibration and noise inside the car, and affect passengers' comfort ${ }^{[7]}$. Currently there are four kinds of engine mounts: rubber mount, hydraulic mount, semi-active hydraulic mount and hydraulic mount. This paper focuses on the semi-active hydraulic engine mount to study on its dynamic characteristics. At present, we have four methods to build up models of the semi-active hydraulic engine mount: pure mathematical modelling in which we need to solve vibration differential equation, lumped parameter modelling combining mechanic and fluid theory, bond graph modelling and finite element modelling ${ }^{[5][6]}$ In this paper, the finite element method is used under the condition of solid-fluid interaction. Solidfluid interaction is intended to study the influence of fluid on a variety of solid deformation, and the flow field under the action of fluid. It emphasizes on interaction between solid and fluid. Solid under the influence of fluid will deform or move. Deformation and movement, in turn, will affect the flow field. So solving solid-fluid interaction is not solving fluid or solid alone. It is a process of mutual iterative calculation.

\section{Structures and Working Principle of Semi-Active Hydraulic Mount}

The sectional view of semi-active hydraulic engine mount's structure is shown in Fig 1, mainly composed of Connecting hole 311, Connecting member 312, Rubber of Main spring 32, Shell of Main spring 33, Cover of Decoupling plate 41, Decoupling membrane 422, Body

\footnotetext{
a Wenku Shi: shiwk@jlu.edu.cn
}

of Throttle plate 43, Inertial track 44, Basement membrane 52, and Basement bowl 53. Among these, Connecting hole, Connecting member and the top of Main spring are connected together by vulcanization. The connecting hole and the engine are joined together to endure motivation from the engine. Inside Shell of Main spring there is a circle raised in the middle part to clamp the main rubber spring. The semi-active control hydraulic mount have a throttle plate, which can increase the liquid damping. Decoupling membrane may ease kinematic hardening to some extent. Main rubber spring is the main part to attenuate vibration. The semi-active hydraulic engine mount studied in this paper was divided into an upper liquid chamber and a lower liquid chamber. Liquid in the upper liquid chamber can flow into the lower liquid chamber through Inertia track, resulting in energy loss to attenuate vibration. There is a solenoid valve attached to a chamber between the bottom of Decoupling membrane and Body of Decoupling plate. When the solenoid valve is open, the air chamber connects to the atmosphere. When the solenoid valve is closed, the air chamber has a certain air pressure, resulting in Decoupling membrane hardening, thus affecting the performance of the mount.

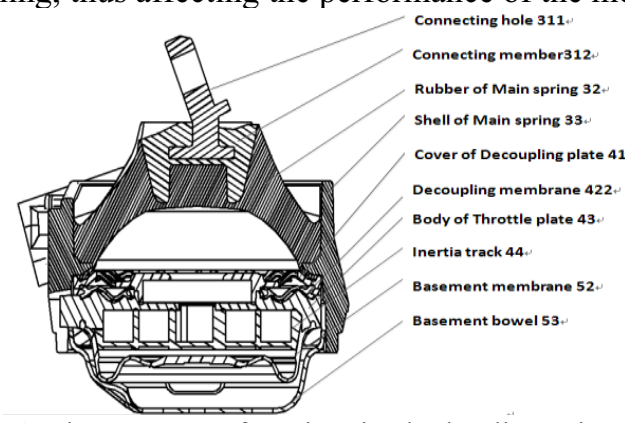

Figure 1. The structure of semi-active hydraulic engine mounts 


\section{Modelling of the Semi-Active Hydraulic Mount}

When using ADINA to simulate the Semi-active Hydraulic Mount, the solid model was built in ADINASTRUCTURE and the solid model was built in ADINACFD. The coupling surface between the solid model and the solid model should correspond to each one. Firstly the three-dimensional model of the Semi-active Hydraulic Mount was meshed in the software HYPERMESH, and then the mesh model was imported in ADINA to set materials, constraints, loads and boundaries. Finally the model was simulated in the solver ADINA-FSI. The result was output-ted from the processing module ADINA-PROCESSING.

\subsection{The Finite Element Model of Solid}

Most of the solid elements are six-sided shape unit, and there are a little of elements are five-sided shape unit where the structure is complex. The numbers of elements of each part are: Main spring 5472, Throttle plate body 12954, Snap ring 1656, Basement membrane 9162, Connecting member 2688, and Decoupling membrane 2808 .

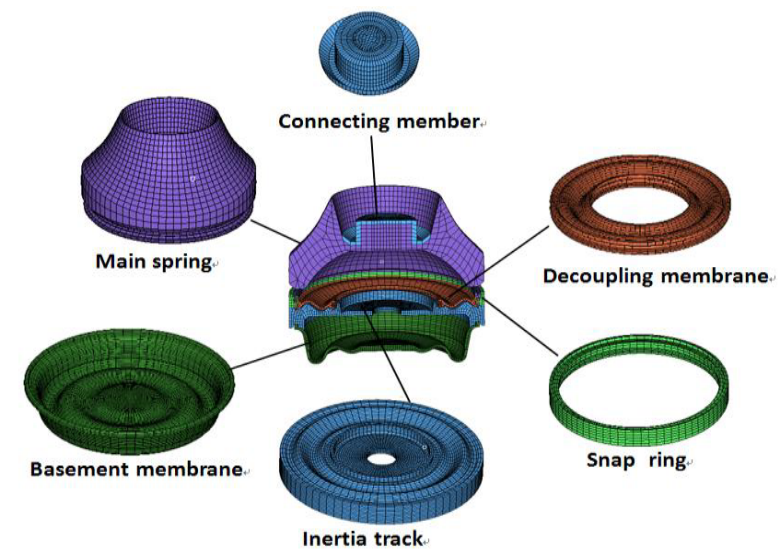

Figure 2. Finite element model

In this Semi-active Hydraulic Engine Mount, the material of Connecting Member is steel, and its parameters are: Density $7.8 \times 10^{-6} \mathrm{~kg} / \mathrm{mm}^{3}$, Poisson ratio 0.29 , Elastic Modulus $2.07 \times 10^{5} \mathrm{~N} / \mathrm{mm}^{2}$.

Main spring is the main isolation part and it is made of rubber, so it is important to get the rubber's parameters. The constitutive models of super-elastic material are divided into two categories: Statistical Thermodynamic Model and Continuum Mechanics Model based on the phenomenological theory ${ }^{[2][4]}$. The uniaxial tensile test was carried out, and the experimental data was used in order to fit the classic hyper elastic constitutive model. We selected the Mooney-Rivlin model as the rubber's hyper elastic model. Its parameters are: $\mathrm{C} 1=0.245$, C2 $=0.087$.

Main spring, Snap ring and Throttle plate body are bonded to withstand the metal jacket's circumferential load. So we set the constraint on nodes attached to the metal jacket. When this Semi-active Hydraulic Engine Mount works, the force of the engine is loaded onto
Connecting Member. So the stimulus was loaded on the centre of Connecting Member during simulation.

\subsection{The Finite Element Model of fluid}

The fluid model is divided into three parts of Upper fluid chamber, Inertia track and Lower fluid chamber. The quality of liquid elements could seriously affect the simulation, so the good quality of elements is required especially where large variations exist. We take type FCBI element as fluid element. Liquid mesh models are shown in Fig.3. Numbers of each model's elements are: Upper fluid chamber 14519, Inertia track 20114, and Lower fluid chamber 11628.

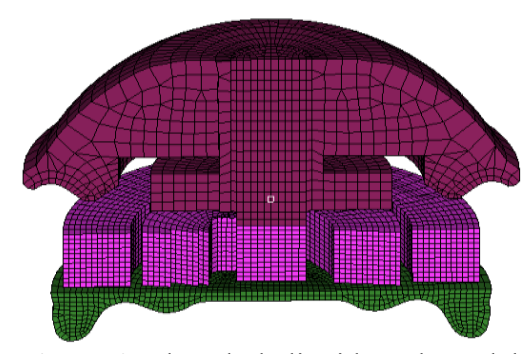

Figure 3. The whole liquid mesh model

In this paper, the suspension liquid is a mixture of $70 \%$ ethylene glycol and $30 \%$ water. Its density is $1.1 \times$ $10^{-9} \mathrm{~T} / \mathrm{mm}$, and the dynamic viscosity is $7.7 \times 10^{-9} \mathrm{MPas}$ $\left(20^{\circ} \mathrm{C}\right)$.

\subsection{The Finite Element Model of gas}

The gas model has the same type of elements with the fluid model. The gas model is shown in Fig.4, and it has 1368 elements. The gas elements and the fluid elements are imputed into ADINA.

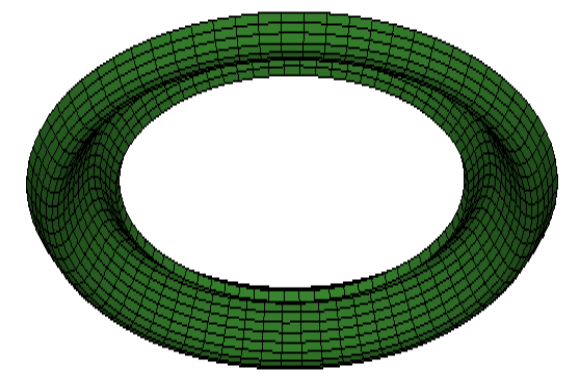

Figure 4. The model of gas

\section{Simulation Analyses and Experimental Verification}

\subsection{Static Characteristics}

The static characteristics include the stiffness of three directions, which were tested by American MTS831 test bench. 


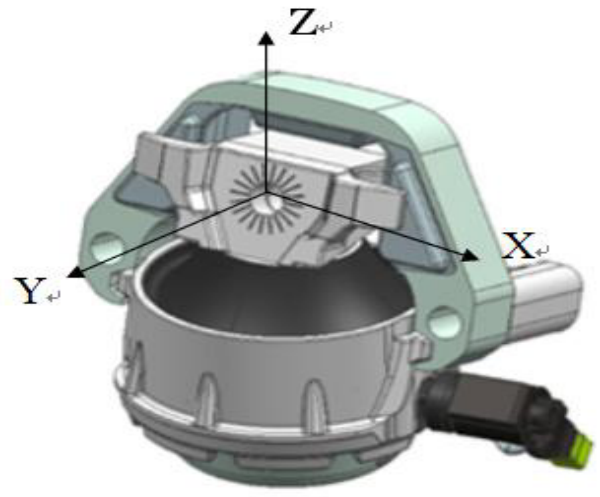

Figure 5. Direction schematic

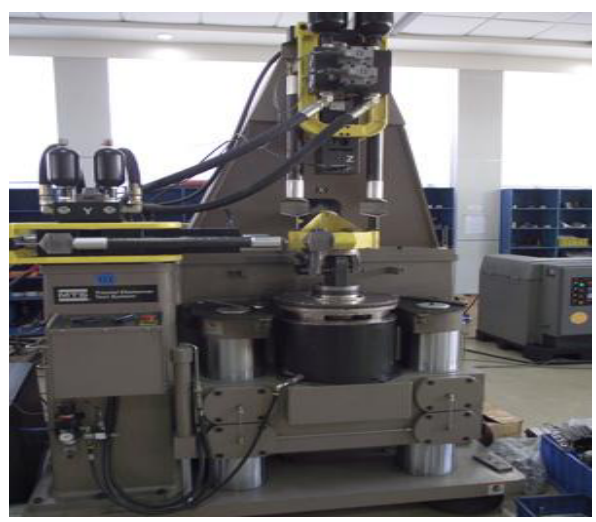

Figure 6. MTS831 test bench

As shown in Table 1, the simulation results of the Semi-active Hydraulic Mount's longitudinal, horizontal and vertical static stiffness were compared with the experimental values. By the simulation error analysis, simulation results were in good agreement with the experimental values. The accuracy of the model was proved initially. Next, the dynamic characteristics of the test results were com-pared with the simulation results to verify the accuracy of the model further.

Table 1. Simulation results and test values of static stiffness.

\begin{tabular}{|c|c|c|c|}
\hline Direction & $\begin{array}{c}\text { Longitudinal } \\
\mathrm{x}\end{array}$ & $\begin{array}{c}\text { Horizontal } \\
\mathrm{y}\end{array}$ & $\begin{array}{c}\text { Vertical } \\
\mathrm{z}\end{array}$ \\
\hline $\begin{array}{c}\text { Vertical preload } \\
(\mathrm{N})\end{array}$ & 950 & 950 & 161 \\
\hline $\begin{array}{c}\text { Simulation results of } \\
\text { static stiffness } \\
(\mathrm{N} / \mathrm{mm})\end{array}$ & 205.6 & 191.1 & 162.5 \\
\hline $\begin{array}{c}\text { Test values of } \\
\text { static stiffness } \\
(\mathrm{N} / \mathrm{mm})\end{array}$ & 208.5 & 192.2 & $0.09 \%$ \\
\hline Error & $1.4 \%$ & $05 \%$ & $0.09 \%$ \\
\hline
\end{tabular}

\subsection{Dynamic Characteristics}

The evaluations of dynamic characteristics include dynamic stiffness and damping angle ${ }^{[1][3]}$. There are two working suspension state in Semi-active Hydraulic Engine Mount: (1) the bottom of the Decoupling membrane is vented to the atmosphere i.e. the solenoid valve is open. (2) The bottom of Decoupled membrane is sealed i.e. the solenoid valve is closed. Test and simulation programs are as follows: preloads are $950 \mathrm{~N}$ and $1100 \mathrm{~N}, 25$ typical frequencies are selected, and the amplitude of sinusoidal load is $0.1 \mathrm{~mm}$.

\subsubsection{The Solenoid Valve Is Open}

When the solenoid valve is open, vertical dynamic characteristics of the test results and simulation results are shown in Fig.7, Fig.8, Fig.9 and Fig.10. As can be seen from the compared curves, simulation curves are in good agreement with the experimental curves. It shows the correctness of the model.

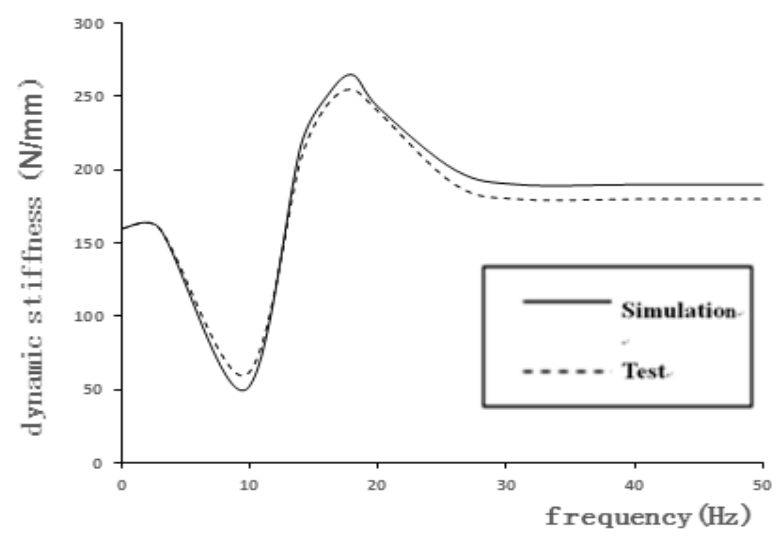

Figure 7. Dynamic stiffness (Valve Open-preload 950N)

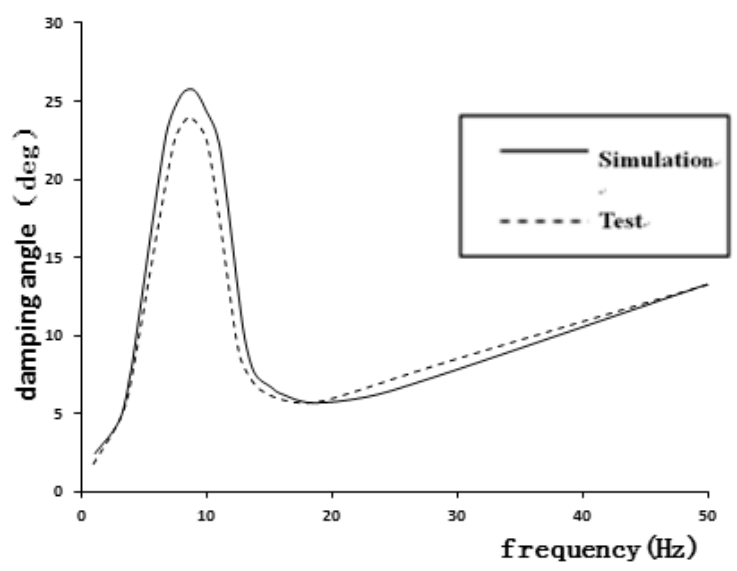

Figure 8. Damping angle (Valve Open-preload 950N)

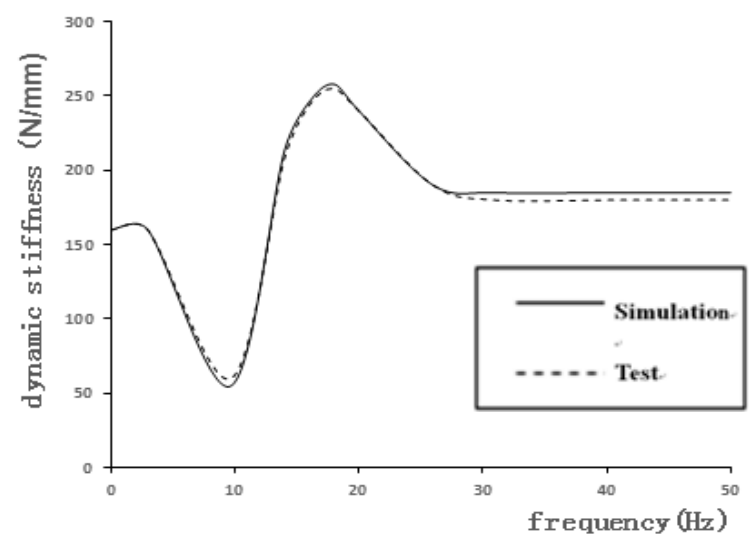

Figure 9. Dynamic stiffness (Valve Open-preload 1100N) 


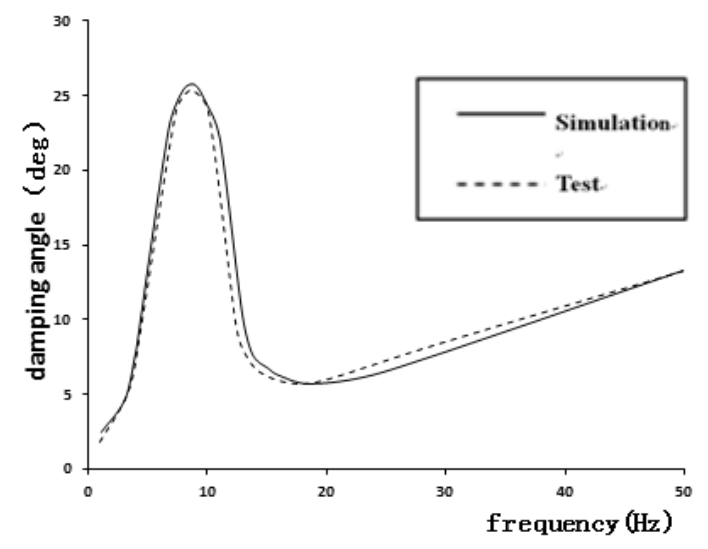

Figure 10. Damping angle (Valve Open-preload 1100N)

\subsubsection{The Solenoid Valve Is Closed}

When the solenoid valve is closed, the damping angle increases, especially at high frequencies. As can be seen from Fig.11, Fig.12, Fig.13, and Fig.14, simulation curves are in good agreement with the experimental curves. It shows the correctness of the model.

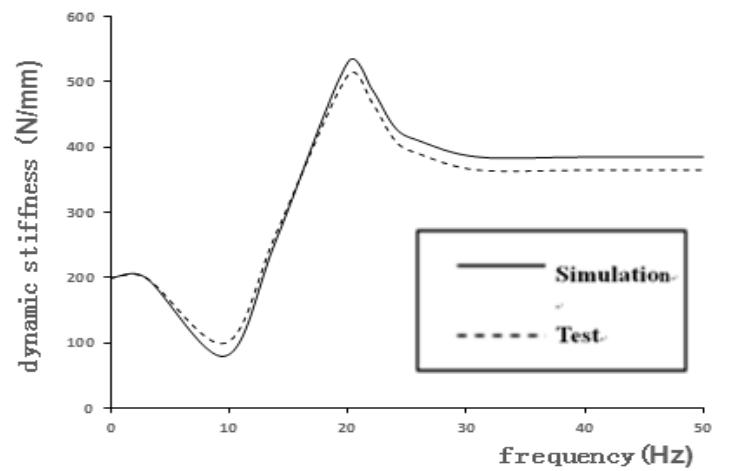

Figure 11. Dynamic stiffness (Valve Closed-preload 950N)

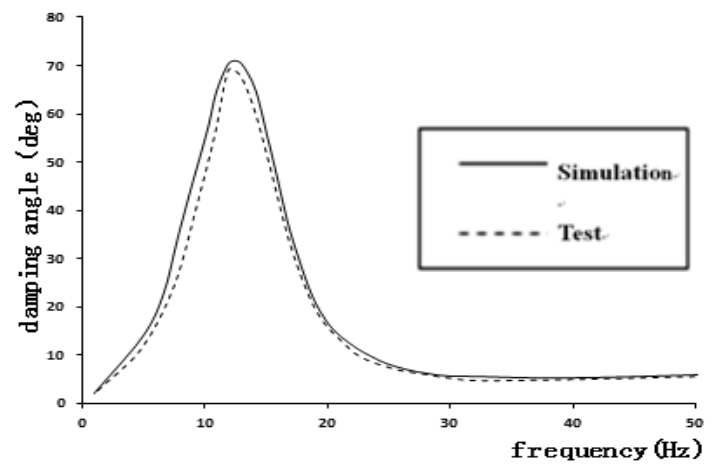

Figure 12. Damping angle (Valve Closed-preload 950N)

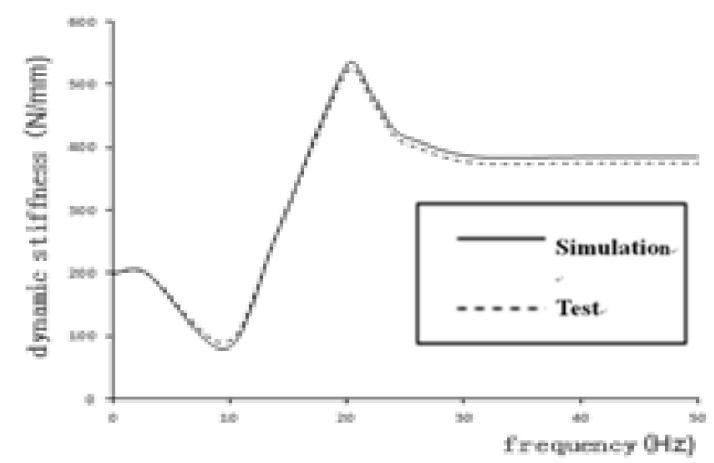

Figure 13. Dynamic stiffness (Valve Closed-preload 1100N)

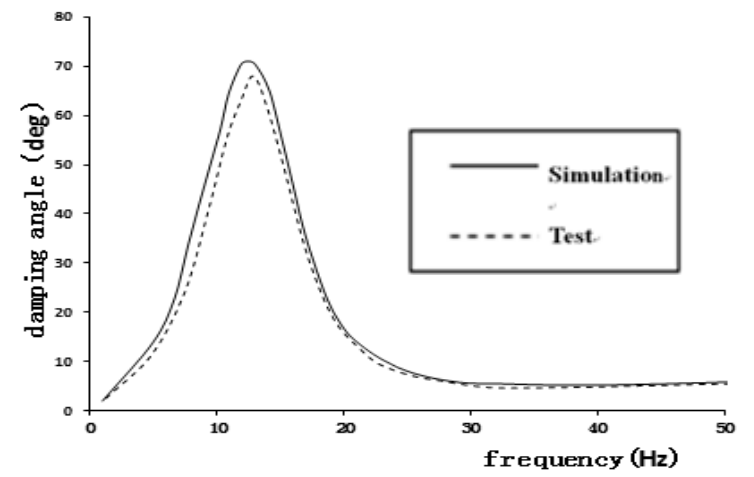

Figure 14. Damping angle (Valve Closed-preload 1100N)

\section{Conclusions}

- The finite element models of solid and fluid were built in ADINA, and the model could intuitively reflect the construction and the interaction between flu-id and solid in the semi-active hydraulic engine mount.

- According to the test, the simulation results in ADINA are in good agreement with the experimental results. The accuracy of the model is verified.

- Based on this model, the parameters could be analysed, which affect the performance of the semi-active hydraulic engine mount greatly.

\section{References}

1. Bo-Ha Lee, Chong-Won Lee. Journal of Sound and Vibration, J. E, 323,(2009)

2. Boyce M C, Arruda E M. Rubber Chemistry and Technoligy, J. E, 73, 3 (2000)

3. Coreoran P., Tieks G. SAE Technical Paper Series, 840407, (1984)

4. LI Xiao-fans, YANG Xiao-xiane. China Elastomerics, 15, 1 (2003)

5. Thanh Quoc Truong, Kyoung Kwan Ahn. Journal of Sound and Vibra-tion J. E, 329,(2010)

6. Vahdati Nader, Saunders L, Ken Lauderbaugh. ISA Transactions, 41, 2(2002)

7. Wallacc C.Flowcr. Vibration and Ride Qualities, SAE Paper, 850975, (1985)

8. Yasuhiro Kakinuma, Tojiro Aoyama, Hidenobu Anzai, et al. Preci-sion Engineering,J. E, 291,(2006) 